\title{
A survey of amphetamine prescribing by drug services in the East and West Midlands
}

\section{AIMS AND METHOD}

This study aimed to assess the current level of amphetamine prescribing for adults in drug services in the West and East Midlands. A questionnaire was developed to investigate prescribing habits and attitudes, and was addressed to the senior member of staff in each drug service within the West and East Midlands area.

\author{
RESULTS \\ A total of 41 services were identified, \\ and 29 questionnaires were returned \\ (a $71 \%$ response rate). Of the services \\ that replied, $20(69 \%)$ prescribed \\ amphetamines, with 132 (12\%) \\ patients identified as amphetamine \\ misusers currently receiving a pre- \\ scription. However, 26 (90\%) services \\ felt that substitute prescribing did \\ have a role in a comprehensive \\ service for this group.
}

\begin{abstract}
CLINICAL IMPLICATIONS
Although amphetamine prescribing has been shown to reduce both criminal and injecting behaviour, only two-thirds of the local services prescribe such drugs, with a small percentage of amphetamine users receiving a prescription. There is a need for a national consensus about substitute prescribing of amphetamines and increased flexibility within drug services when managing the problem.
\end{abstract}

Amphetamines are the second commonest misused drug in the UK, particularly among younger people (DrugScope, 2000), and there is some evidence that the problem has increased considerably in recent years. Fleming and Roberts (1994) reported a 175\% increase in amphetamine use between 1990 and 1992, with over $60 \%$ injecting. Other studies of amphetamine users appearing at needle exchanges also suggest a large unmet need for treatment (Seivewright \& McMahon, 1996).

Historically, psychostimulants have been proposed to have a role in treating a number of psychiatric disorders, including depressive disorders, adult attentiondeficit hyperactivity disorder (ADHD) and narcolepsy (Beck et al, 1999). Furthermore, there is some evidence that amphetamine prescribing for drug misusers is effective in reducing injecting and criminal behaviour (Fleming \& Roberts, 1994; Charnaud \& Griffiths, 1998). However, the prescription of psychostimulants for amphetamine misusers remains controversial. Many clinicians are reluctant to prescribe them, partly because their use is not licensed for the treatment of drug dependence. Furthermore, there are a number of potential disadvantages including precipitation of episodes of psychosis and intracerebral haemorrhage (McEvoy et al, 2000) and as yet there is no national consensus or endorsement of this practice (Department of Health, 1999).

This study aimed to assess the current level of amphetamine prescribing for adults by drug services in the East and West Midlands. In addition we aimed to survey the views of the staff working in drug services concerning the desirability of prescribing amphetamines.

\section{Method}

A postal survey of all registered drug services in the East and West Midlands was carried out between November 1999 and May 2000. All statutory and non-statutory drug services were identified through the 1999 UK AIDS Directory (National Aids Manual, 1999). A simple questionnaire was developed and addressed to the senior staff of each service, accompanied by a covering letter and a stamped addressed envelope.

The survey asked the service whether it had prescribed amphetamines to patients in the past 6 months, and the number of amphetamine misusers that had used the replacement prescribing during this period. Further questions aimed to determine the availability of a prescribing doctor and his/her feelings regarding replacement amphetamine prescribing, as well as any inclusion or exclusion criteria set down in order to receive such a service. The attitudes of the service as a whole towards prescribing amphetamines and the possibility of receiving other modalities of intervention were also clarified.

\section{Results}

A total of 41 services were identified and sent questionnaires. Two mailings resulted in 29 completed forms, a response rate of $71 \%$. Three services $(10 \%)$ identified themselves as addiction treatment units, $18(62 \%)$ as community drug teams, six (21\%) as non-statutory drug services and two (7\%) as combined community drug team and drug treatment units. Nineteen services had a doctor attached who was responsible for assessment and prescribing.

\section{Reasons for prescribing}

Twenty of the 29 services (69\%) prescribed amphetamines, and a variety of reasons were cited. Nineteen $(66 \%)$ stated that they had prescribed them as a substitution for illicit amphetamine use, three (10\%) for ADHD, nine (31\%) because amphetamine misuse had led to 
original papers
Table 1. Variety between services of inclusion criteria for

amphetamine prescribing

\begin{tabular}{lc}
\hline Inclusion criteria & $\begin{array}{c}\text { Number (\%) } \\
(n=20)\end{array}$ \\
\hline $\begin{array}{l}\text { Evidence of daily use } \\
\text { Evidence of injecting }\end{array}$ & $6(30)$ \\
To reduce the harm of viral infections, legal & $5(25)$ \\
$\quad$ problems or mental illness & $5(25)$ \\
Amphetamine use for 3 years & $1(5)$ \\
As an antidepressant & $1(5)$ \\
As a last resort & $2(10)$ \\
\hline
\end{tabular}

social problems, eight (28\%) for psychological or medical problems and eight (28\%) for legal problems.

Inclusion criteria for amphetamine prescribing varied between services and are listed in Table 1. Exclusion criteria included cardiac problems, high blood pressure, mental illness, paranoid symptoms, violence and evidence of leakage onto the 'black market'.

\section{Current service users}

The services surveyed estimated that they had seen a total of 6584 patients in the previous 6 months, with the number seen by each service ranging from 60-1200. The corresponding figure for amphetamine misusers was 1120 , with each service having contact with between two and 300 patients. The number of patients who were prescribed amphetamine within each service ranged from one to 40 , with a total of 132 across the 29 services. Thus, the group-prescribed amphetamines made up only $2 \%$ of all patients attending the respondents' services. Three (10\%) services prescribed the amphetamines in the form of tablets only and a further $10(35 \%)$ used only elixir, with seven (24\%) using a combination of both forms. No services prescribed injectable amphetamines.

\section{Attitudes towards amphetamine prescribing}

Twenty services (69\%) felt that amphetamine prescribing should be restricted to specialist services, and 20 (69\%) stated that it should be time-limited. A maximum dose was felt to be appropriate by 18 services $(62 \%)$, with the suggested maximum dose range between $30 \mathrm{mg}$ and $300 \mathrm{mg}$ daily. However, three services (10\%) felt that the maximum dose should depend on assessment of individual needs. The majority of services $(26 ; 90 \%)$ felt that amphetamine substitute prescribing should be available in specific cases (as determined by their inclusion criteria), with only three $(10 \%)$ emphasising that treatment for amphetamine using patients should only include counselling and social interventions.

\section{Discussion}

This was the first local survey of current practice and attitudes toward prescribing amphetamines, and the aim was to examine this issue from the perspective of drug service providers. As with all questionnaire surveys there were a number of limitations. Services offering substitute prescribing to amphetamine users may have been more likely to return the survey, and a number of the services included had no prescribing role. Each service estimated the total number of patient contacts in the previous 6 months, but some gave more accurate figures than others. However, we believe that the percentage of amphetamine users that received substitute prescribing was broadly correct.

The study demonstrated a significant level of disparity between beliefs and practice in the area of treating amphetamine misuse. Approximately $90 \%$ of the services questioned felt that substitute prescribing should be available in addition to psychological and social interventions as part of a comprehensive service for this group. However, only two-thirds (69\%) of the services actually prescribed amphetamines, and relatively few patients identified as having problems with amphetamines actually received substitution therapy (12\%). There is a slowly developing body of research evidence for the use of substitute prescribing, and many feel that it is a potential method of engaging an often neglected group of patients in treatment (Klee \& Morris, 1995). Furthermore, there is some evidence that it reduces injecting in up to $70 \%$ of amphetamine misusers (Charnaud \& Griffiths, 1998). Thus there is clearly a need for consensus in this area, backed up by well-designed research studies.

\section{References}

BECK, C., SILVERSTONE, P., GLOR, K., et as a harm reduction measure? Journal al (1999) Psychostimulant prescriptions of the Royal Society of Health, 114, by psychiatrists higher than expected: a self-report survey. Canadian Journal of Psychiatry, 44, 680-684. 127-131.

KLEE, H. \& MORRIS, J. (1995) The role of needle exchange in modifying sharing behaviour: cross study comparisons 1989-1993. Addiction, 90, 1635Levels of intravenous drug misuse among clients prescribed oral dexamphetamine or oral methadone: a comparison. Drug and Alcohol Dependence, 52,79-84.

DEPARTMENT OF HEALTH (1999) Drug Misuse and Dependence - Guidelines on Clinical Management. London: Department of Health.

DRUGSCOPE (2000) UK Drug Situation 2000. The UK report to the European Monitoring Centre for Drugs and Drug Addiction. London: DrugScope.

FLEMING, P. M. \& ROBERTS, D. (1994) Is prescription of amphetamine justified 1645

MCEVOY, A.W., KITCHEN, N. D. \& THOMAS, D. G. (2000) Intracerebra haemorrhage in young adults: the emerging importance of drug misuse. BMJ, 320, 1322-1324

NATIONAL AIDS MANUAL (1999) UK AIDS Directory 1999-2000. London: Nam Publishing.

SEIVEWRIGHT, N. \& MCMAHON, C. (1996) Misuse of amphetamines and related drugs. Advances in Psychiatric Treatment, 2, 211-218.

*Hamdy F. Moselhy Consultant Psychiatrist, George Georgiou Consultant Psychiatrist, Ash Kahn Consultant Psychiatrist, Ed Day Specialist Registrar, Addictive Behaviours Centre, Northern Birmingham Mental Health NHS Trust, 71 Fentham Road, Erdington, Birmingham B23 6AL 